\title{
Sleep patterns among patients with chronic fatigue: A polysomnography-based study
}

\section{Evelina Pajediene' (D) | Indre Bileviciute-Ljungar ${ }^{2,3,4}$ | Danielle Friberg5}

\author{
${ }^{1}$ Department of Neurology, Kaunas \\ Clinics, Hospital of Lithuanian University \\ of Health Sciences, Kaunas, Lithuania \\ ${ }^{2}$ Department of Clinical Sciences, \\ Karolinska Institutet, Stockholm, Sweden \\ ${ }^{3}$ Department of Rehabilitation Medicine, \\ Danderyd University Hospital, Stockholm, \\ Sweden \\ ${ }^{4}$ Department of Medical and Health \\ Sciences, Faculty of Medicine and Health \\ Sciences, Linköping University, Pain and \\ Rehabilitation Centre, Anaesthetics, \\ Operations and Specialty Surgery Centre, \\ Region Östergotland, Linköping, Sweden \\ ${ }^{5}$ Department of Clinical Science, \\ Intervention and Technology (CLINTEC), \\ Division of Otorhinolaryngology, \\ Karolinska University Hospital, \\ Stockholm, Sweden

\section{Correspondence} \\ Evelina Pajediene, MD, Resident in \\ Neurology, Department of Neurology, \\ Kaunas Clinics, Hospital of Lithuanian \\ University of Health Sciences, Eiveniu St 2, \\ Kaunas 50009, Lithuania. \\ Email: evelinaprei@gmail.com
}

\begin{abstract}
Objectives: The purpose of this study was to detect treatable sleep disorders among patients complaining of chronic fatigue by using sleep questionnaires and polysomnography.

Methods: Patients were referred to hospital for investigations and rehabilitation because of a suspected diagnosis of myalgic encephalomyelitis/chronic fatigue syndrome (ME/CFS). The criteria for further referral to full-night polysomnography (PSG) were symptoms of excessive daytime sleepiness and/or tiredness in the questionnaires.
\end{abstract}

Results: Of a total of 381 patients, 78 (20.5\%) patients underwent PSG: 66 women and 12 men, mean age 48.6 years, standard deviation \pm 9.9 years. On the basis of the PSG, 31 (40.3\%) patients were diagnosed with obstructive sleep apnoea, 7 (8.9\%) patients with periodic limb movement disorder, 32 (41.0\%) patients with restless legs syndrome and 54 (69.3\%) patients had one or more other sleep disorder. All patients were grouped into those who fulfilled the diagnostic criteria for ME/CFS $(n=55$, $70.5 \%)$ and those who did not $(n=23,29.5 \%)$. The latter group had significantly higher respiratory $(P=.01)$ and total arousal $(P=.009)$ indexes and a higher oxygen desaturation index $(P=.009)$.

Conclusions: More than half of these chronic fatigue patients, who also have excessive daytime sleepiness and/or tiredness, were diagnosed with sleep disorders such as obstructive sleep apnoea, periodic limb movement disorder and/or restless legs syndrome. Patients with such complaints should undergo polysomnography, fill in questionnaires and be offered treatment for sleep disorders before the diagnose ME/ CFS is set.

\section{K E Y W O R D S}

chronic fatigue syndrome, fatigue, myalgic encephalomyelitis, polysomnography, sleep, sleep apnoea

\section{1 | INTRODUCTION}

Many patients who consult doctors from a variety of medical specialties complain about feeling tired, sleepy or fatigued, which is causing a significant economic burden at both the individual and societal level. ${ }^{1}$ The process of diagnosing a fatigued patient should begin with clearly defining the symptoms, before proceeding to the exclusion of sleep-related and psychosomatic comorbidities.
Myalgic encephalomyelitis/chronic fatigue syndrome (ME/CFS) is characterized by physical and mental fatigue and fatigability that are not ameliorated by rest. In addition, patients suffer from widespread diffuse pain and cognitive difficulties that last more than 6 months. ${ }^{2}$ Patients often report malaise, worsening of symptoms with effort, non-restorative sleep, sore throat, swollen lymph nodes and other symptoms. ${ }^{3}$ Depending on the criteria used, the prevalence varies from $1.2 \%$, when the more strict 
Canadian criteria are applied, to $2.6 \%$, when the broad Oxford criteria are used. ${ }^{4}$

Even though in all available case definition guidelines of CFS, sleep problems are described as a minor criterion, ${ }^{5}$ the exact definition of sleep disturbance is not clear. According to a phone survey-based study by Unger et al, one of the most prevalent complaints among patients diagnosed with CFS is unrefreshing sleep; however, this study did not find any increased association between subjectively expressed chronic fatigue and excessive daytime sleepiness or obstructive sleep apnea (OSA). ${ }^{6}$ According to several studies, the undiagnosed primary sleep disorder was detected in $0-50 \%$ of chronically fatigued patients. ${ }^{7,8}$ Through a detailed diagnostic investigation with questionnaires, polysomnography (PSG) and multiple sleep latency testing, Mariman et al found that $49.8 \%$ of patients diagnosed with ME/CFS were suffering from predominant or co-morbid sleep disorders and that $28.7 \%$ had OSA. ${ }^{9}$ A PSG-based controlled study by Neu et al detected more slow-wave sleep and microarousals in 16 CFS patients, but also lower grip strength and reduced concentrations of circulating cytokines. However, the group did not find OSA or periodic limb movement disorder (PLMD). ${ }^{10}$

In the present study, we hypothesized that chronically fatigued patients with excessive daytime sleepiness and/or tiredness suffer from treatable sleep-related disorders such as OSA, PLMD and restless legs syndrome (RLS), which could be detected by using PSG and specific questionnaires.

\section{2 | MATERIALS AND METHODS}

\section{1 | Participants}

The participants were consecutive patients who entered the ME/CFS project at Danderyd University Hospital, Stockholm in the period from 2011 to 2013. The unit in Danderyd University Hospital served as a public healthcare tertiary clinic for patients with chronic fatigue. The majority of patients were referred from primary health care clinics and psychiatry and neurology departments within Stockholm County.

A clinician, who was a specialist in rehabilitation medicine or neurology, screened every patient over the course of a 90-minute consultation. Investigations with laboratory tests were performed in order to exclude ongoing inflammation, infection, metabolic and immunological disorders and/or other pathological conditions. In a majority of patients, brain magnetic resonance imaging was performed in order to exclude brain disorders. In addition, patients met a certified psychologist for 90 minutes of psychological evaluation including a standardized psychiatric interview and neuropsychological testing. The diagnosis of ME/CFS was made in accordance with the Centers for Disease Control $^{2}$ and/or Canadian criteria ${ }^{3}$ following medical, psychological, laboratory results, brain imaging and PSG investigation/treatment of OSA.

Our inclusion criteria for performing PSG for all consecutively referred patients were symptoms of excessive daytime sleepiness, by using Epworth sleepiness scale $\geq 10$, and/ or often or always tired during mornings and/or daytime, from specific questionnaires (see below). In-lab, full-night PSG was performed at the Department of Otorhinolaryngology (ORL), Karolinska University Hospital (see below). Afterward, the results of PSG and all related sleep complaints were processed by a specialist in sleep medicine (DF).

Following these evaluations, including PSG, all patients were divided into two groups: those diagnosed with ME/ CFS (the ME/CFS group) and those diagnosed with other disorders (the non-ME/CFS group). The group of non-ME/ CFS patients did not fulfil the symptom-based criteria for ME/CFS or had exclusionary conditions, such as psychiatric disorders (untreated burn-out syndrome, depression and/or anxiety) often together with pain syndrome, or a suspected exclusionary or untreated somatic disorder that could explain the symptoms. The strict exclusionary criteria regarding obesity and drug abuse were applied before accepting a referral because of official referring guidelines. Referrals were discussed by two CFS-specialists before approval.

If a diagnosis of OSA and/or PLMD was revealed on the basis of the PSG, the patient was offered treatment and follow-up. Patients with mild sleep disturbances were provided with treatment recommendations through their primary care clinic, while those with moderate-to-severe OSA were followed-up by the sleep specialist (DF). If PLMD and/or RLS were diagnosed, the patients were referred to a neurologist. However, the results of treatment are not presented, as treatment outcomes were not within the scope of this study.

\section{2 | Questionnaires}

1. The Epworth Sleepiness Scale (ESS)—a self-administered questionnaire measuring the subject's general level of daytime sleepiness. ${ }^{11}$

2. The ORL department's local self-reported sleep questionnaire consisting of six questions about OSA symptoms such as snoring, breathing pauses during sleep, sleepiness while driving and morning and daytime tiredness. There were also five questions concerning insomnia-related complaints. A four-grade Likert scale with the alternatives never, sometimes, often, or always was used.

3. A validated questionnaire concerning RLS with four positive responses being mandatory for the diagnosis of RLS. $^{12}$ 
4. A validated question concerning self-rated health with five options, from 'excellent' to 'very poor'. ${ }^{13}$

5. The Swedish version of the Multidimensional Fatigue Inventory (MFI-20) covers five dimensions of fatigue: general fatigue, physical fatigue, mental fatigue, reduced motivation and reduced activity. ${ }^{14}$

6. The Hospital Anxiety and Depression Scale (HADS)used to determine the levels of anxiety and depression. ${ }^{15}$

\section{3 | Polysomnography}

In-lab, full-night PSG was performed using the Embla technology (Flaga Medical; Reykjavik, Iceland). Measurements were interpreted manually by registered technologists and checked by a specialist in sleep medicine (DF). The patients were woken at 6 am because of the sleep laboratory being located in a day-care unit. Sixteen channels were recorded: EEG (sensors C3-A2, O1-A2, O2-A1 and C4-A1), EOG (left and right), EMG chin and tibialis (left and right), oronasal flowmetry, respiratory movements (abdomen and thorax), snoring, ECG, pulse and body position. Parameters were defined in accordance with American Academy of Sleep Medicine 2007, using criteria B for the hypopnoea, that is, at least a $50 \%$ flow reduction leading to either a 3\% oxygen desaturation or arousal. ${ }^{16}$ For mild OSA diagnose, the RDI is $\geq 5$, for moderate OSA, the RDI is $\geq 15$ and, for severe OSA, the RDI is $\geq 30$. The primary outcome in the present study was the RDI value. The other used respiratory parameters were the Apnea-Hypopnea Index (AHI) and Respiratory Effort-related arousals (RERA), which added together defines the RDI-value. Also, the oxygen desaturation index (ODI) of $3 \%$ or more was calculated. Periodic limb movements (PLM) and those causing arousals (PLMAI) were also noted and periodic limb movement disorder (PLMD) was diagnosed if the PLMAI was equal or higher than five.

Medications regularly used by the patients were not withdrawn prior to the PSG or in the night when PSG was performed. The patients responded to a questionnaire containing questions about whether they had taken any sleeping pills, if the night was normal and if there were something that had bothered them. The patients were woken at $6 \mathrm{am}$.

The use of clinical data for scientific analysis was considered by the regional ethical review board in Stockholm (Ref. no. 2014/300-31) and approved by Danderyd University Hospital (DS2014-0447).

\subsection{Statistical analysis}

The Shapiro-Wilk test was used to test the normality of the sample when the hypothesis of normality is rejected. Descriptive statistics is represented by percentages for qualitative variables and by means and standard deviations for quantitative variables.

The ME/CFS group and the non-ME/CFS group were compared using the Mann-Whitney $U$ test or chi-squared test for unpaired data and the nonparametric parameters and the $t$-test for the parametric parameters. Pearson correlation was used to analyse correlations between two parametric quantitative variables, while Spearman correlation was used to analyse correlations between qualitative variables, or if at least one of them was nonparametric. Ninety-five percent confidence intervals (95\% CIs) for proportions were calculated. A $P$-value of less than .05 was considered to be significant. The statistical package SPSS 17.0 and MS Excel were used for coding and analysing the data.

\section{3 | RESULTS}

\section{1 | Participants}

Patient flow descriptions and changes are presented in Figure 1. A total of 381 patients entered the ME/CFS project, 78 (20.5\%) patients fulfilled the inclusion criteria for performing PSG. Following PSG, 55 (70.5\%) patients were diagnosed with ME/CFS and 23 (29.5\%) patients were diagnosed with other disorders (Figure 1). The 78 participants consisted of 66 women and 12 men with a mean age of 48.6 years, $\mathrm{SD} \pm 9.9$ years. All demographic characteristics and information about the usage of medications that affect sleep are presented in Table 1.

\section{2 | Subjective sleepiness, tiredness, self- rated health, fatigue and emotional status}

The mean ESS score was 9.3 (SD \pm 5.9$)(0-23)$ points and there were $34(43.6 \%)$ patients with a pathological ESS $\geq 10$. When asked about how often they had daytime sleepiness, 68 (87.2\%) patients reported feeling this sensation almost every day.

According to the sleep questionnaire, the majority of respondents (94.9\%) complained about being often or always tired, both in the morning and during the daytime (Table 2). The most frequent OSA-related symptom was snoring (14 patients, $17.9 \%$ ), but apneas during sleep were often or always observed by only two patients, or $2.6 \%$ of the entire study group.

The majority of respondents $(n=75,96.2 \%)$ evaluated their self-rated health status as 'quite poor' or 'very poor'. The emotional status of all 78 (100\%) patients was evaluated using the HADS; with a mean depression index of $7.9 \pm 4.5$ $(1-20)$ and $25(32 \%)$ patients scored $\geq 10$. The mean anxiety index was $6.2 \pm 4.8(0-21)$ and $18(23.1 \%)$ patients scored $\geq 10$. Different components of fatigue experienced by 69 


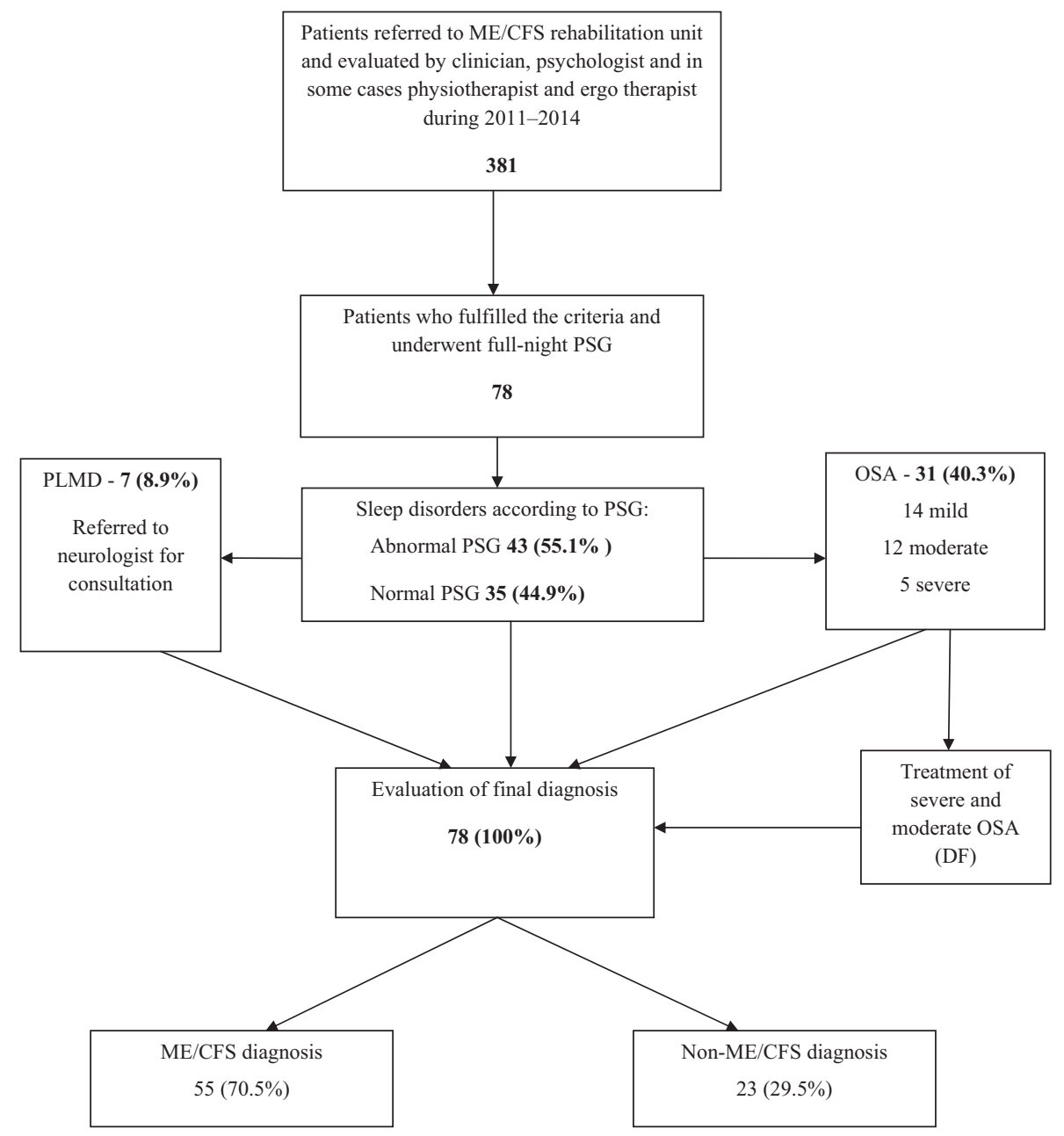

FIGURE 1 Represents the flow chart of the study

(88.5\%) patients were evaluated using the MFI-20 questionnaire; with a mean score for general fatigue of $19.4 \pm 1.7$ (11-20), for physical fatigue of $18.9 \pm 1.8$ (12-20), for reduced physical activity of $17.9 \pm 2.7$ (9-20), for reduced motivation of $10.5 \pm 4.2$ (4-20) and for mental fatigue of $15.7 \pm 3.0$ (9-20). These MFI-20 subscale values were elevated in comparison to normal values in the healthy Swedish population. ${ }^{17}$

On the basis of having provided four positive responses to questions about RLS symptoms, this syndrome was diagnosed in almost half of the study group ( $n=32,41.0 \%)$.

\section{3 | Objective sleep measures from PSG}

Table 3 summarizes all sleep measures from the PSG and compares these with normal values. A total of 14 patients had mild OSA, 12 patients had moderate and 5 patients had severe. Seven (8.9\%) patients were diagnosed with PLMD. OSA together with PLMD was detected in four (5.1\%) patients.

Four patients had a total sleep time of less than four hours. The majority of patients $(n=75,96.2 \%)$ slept less than 7 hours and had decreased sleep efficiency $(n=47$, $60.3 \%)$. For more than half of the study group $(n=44$, $57.1 \%$ ), the sleep onset ( $>30$ minutes) was increased.

\subsection{Combined sleep disorders and their relationship to a primary diagnosis of ME/CFS}

On the basis of both the PSG data and the questionnaires, RLS together with PLMD was found in one $(1.3 \%)$ case, RLS together with OSA in $12(15.4 \%)$ and RLS together with OSA and PLMD in three (3.9\%) cases. A total of 54 (69.3\%) patients had one or more diagnosis of a sleep disorder (OSA, PLMD and RLS) (Table 4).

When comparing our two groups of patients, that is, those diagnosed with OSA and/or PLMD and the rest of the patients (diagnosed with RLS or with a normal PSG), no significant difference was found in their ESS, MFI-20 subscales, HADS, self-rated health status and complaints about daytime or morning tiredness.

Patients complaining of chronic fatigue were given either a diagnosis of ME/CFS $(n=55,70.5 \%)$ or another diagnosis 
TABLE 1 Demographic characteristics of the study group

\begin{tabular}{|c|c|}
\hline Characteristic & $\begin{array}{l}\text { Number of patients } \\
\text { (\%) or mean } \pm \text { SD } \\
\text { (min-max) }\end{array}$ \\
\hline $\operatorname{Sex}(n=78)$ & $\begin{array}{l}12(15.4 \%) \text { male, } \\
66(84.6 \%) \text { female }\end{array}$ \\
\hline Age $(n=78)$ & $48.6 \pm 9.9(23-66)$ \\
\hline $\mathrm{BMI}(n=72)$ & $25.4 \pm 4.5(17.6-38.7)$ \\
\hline Living alone $(n=76)$ & $20(26.3 \%)$ \\
\hline Employed $(n=78)$ & $74(94.9 \%)$ \\
\hline Pensioner $(n=78)$ & $2(2.6 \%)$ \\
\hline Student $(n=78)$ & $3(3.9 \%)$ \\
\hline On sick leave $(n=75)$ & $55(73.3 \%)$ \\
\hline Driving a car $(n=74)$ & $46(62.2 \%)$ \\
\hline Smoking $(n=76)$ & $10(13.2 \%)$ \\
\hline $\begin{array}{l}\text { Using alcohol more than } \\
3 \text { days/week }(n=76)\end{array}$ & $3(3.9 \%)$ \\
\hline Sleep affecting medications: & $44(56.4 \%)$ \\
\hline 1. Benzodiazepines & 1. $1(1.3 \%)$ \\
\hline 2. $\mathrm{Z}$ drugs (zolpidem, zopiclone) & 2. $3(3.8 \%)$ \\
\hline 3. Antidepressants & 3. $15(19.2 \%)$ \\
\hline 4. Antihistamines & 4. $2(2.6 \%)$ \\
\hline 5. Antiepileptic drugs & $5.3(3.8 \%)$ \\
\hline 6. Opioids & 6. $1(1.3 \%)$ \\
\hline $\begin{array}{l}\text { 7. More than one of the types } \\
\text { of medication mentioned above }\end{array}$ & 7. $19(24.4 \%)$ \\
\hline
\end{tabular}

(non-ME/CFS group) $(n=23,29.5 \%)$. Table 4 shows the distribution of sleep disorders diagnosed on the basis of the PSG and questionnaire data among these groups. The prevalence of PLMD, suspected insomnia or RLS did not differ significantly between the ME/CFS and non-ME/CFS groups $(P=.29$, chi-square $=7.1)$.

The non-ME/CFS patient group had significantly higher respiratory $(P=.01, t=-2.6)$ and total arousal $(P=.009$, $t=-2.7)$ indexes, higher ODI $(P=.009, Z=-2.6)$, as well as higher RDI $(P=.09, Z=-1.7)$ and AHI $(P=.09$, $Z=-1.7)$ values, compared with the ME/CFS group. However, the OSA rate did not differ significantly between these two groups. There were no significant differences between groups in the comparison of the ESS scores and the rest of the PSG measures.

\section{4 | DISCUSSION}

The main finding of the present study is that nearly half of our study population were diagnosed with a treatable sleep
TABLE 2 Frequency of the sleep-related complaints (marked as 'often' or 'always')

$\begin{array}{ll}\text { Sleep-related complaints } & \begin{array}{l}\text { Number of } \\ \text { patients (\%) }\end{array} \\ \text { OSAS-related complaints } & \\ \text { 1. Snoring } & 1.14(17.9) \\ \text { 2. Snoring as a social problem } & 2.2(2.6) \\ \text { 3. Breathing pauses during sleep } & 3.2(2.6) \\ \text { 4. Tiredness in the morning } & 4.74(94.9) \\ \text { 5. Tiredness during the daytime } & 5.74(94.9) \\ \text { 6. Nod off at the wheel } & 6.5(6.4)\end{array}$

Insomnia related complaints

1. Insomnia attacks

1. $6.9(11.5)$

2. Awakenings during the night

2. $47(60.3)$

3. Problems falling asleep

3. $32(41.0)$

after awakenings

4. Problems falling asleep

4. $31(39.7)$

at the beginning of the night

5. Less than $6 \mathrm{~h}$ of sleep

5. $14(17.9)$

Sleep related movement disorders complaints

1. Periodic limb movements

1. $6(7.7)$

2. RLS (four positive answers

2. $32(41)$

to questions about RLS symptoms)

3. Patients with both PLM and

3. $15(19.2)$

RLS complaints

Sleepiness-related complaints

1. ESS $\geq 10$

1. $34(43.6)$

2. Daytime sleepiness

2. $68(87.2)$

(almost every day)

Self-rated health evaluated as

$75(96.2)$

'quite poor' or 'very poor'

Data summarized from sleep questionnaire $(n=78)$.

Abbreviations: OSAS, obstructive sleep apnoea syndrome; RLS, restless legs syndrome; PLM, Periodic Limb Movements; ESS, Epworth Sleepiness Scale.

disorder such as OSA (40.3\%) or PLMD (8.9\%) on the basis of the PSG. Forty-one percent had symptoms of RLS, and $69.3 \%$ of patients were diagnosed with one or more sleep disorder (OSA, PLMD and/or RLS). However, there were no significant differences between the groups of ME/CFS and non-ME/CFS patients in terms of the PSG-based diagnosis. These findings are compatible with Le Bon and colleagues study, stating that CFS patients without primary sleep disorders and objective sleepiness could not be distinguished clinically from those diagnosed with a primary sleep disorder or sleepiness. ${ }^{18}$ Moreover, our results are consistent with the study conducted by Mariman et al in which $49.8 \%$ of participants suspected of suffering from ME/CFS were diagnosed with predominant or co-morbid sleep disorders, with an incidence of OSA of $28.7 \% .^{9}$ 
TABLE 3 Sleep measures from the PSGs of all patients, presented as median, mean, standard deviation, minimal and maximal values $(n=78)$

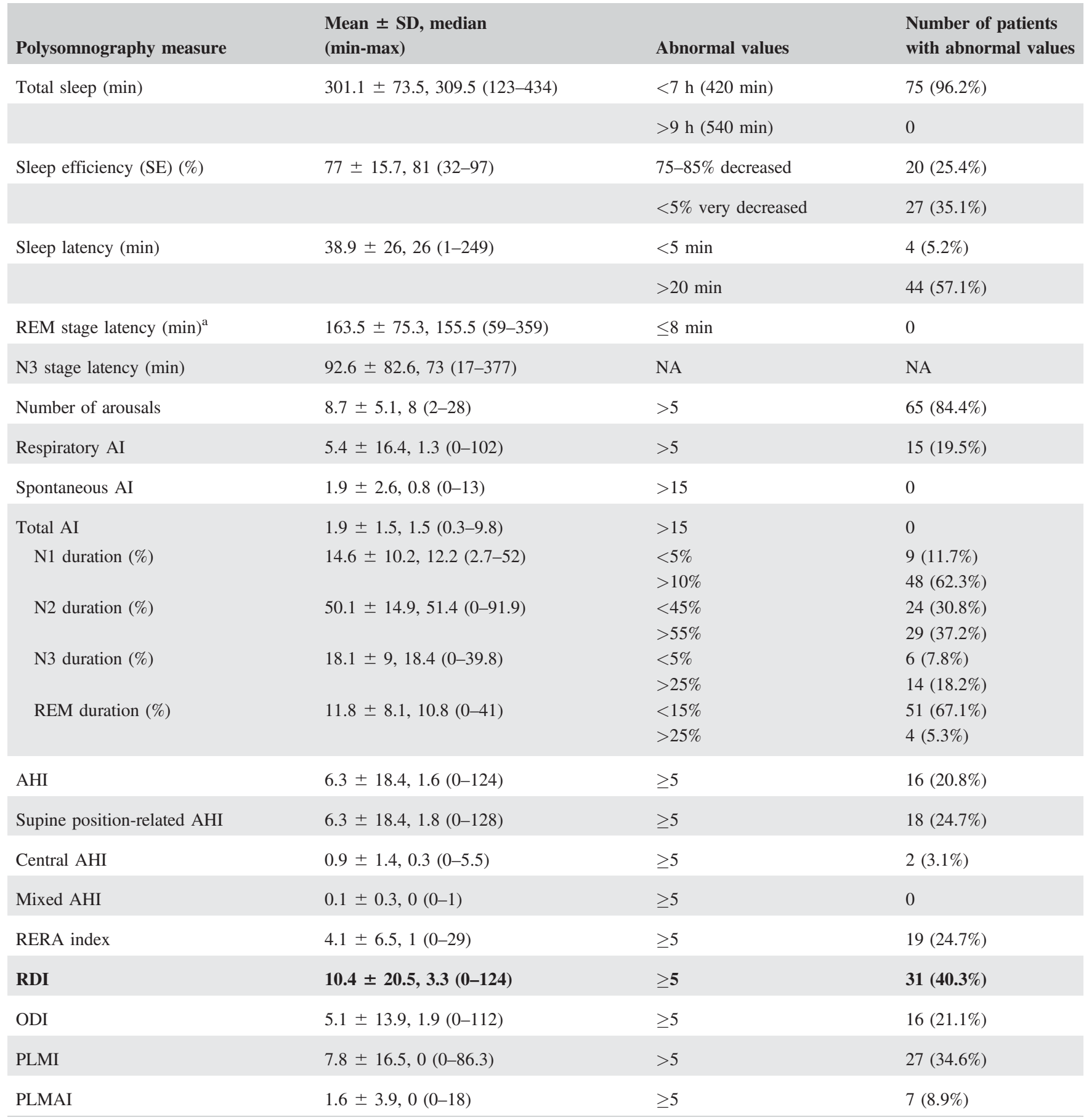

The PSG results are compared with normal values according to the AASM Manual for the Scoring of Sleep and Associated Events (2007). The numbers of patients with abnormal values are presented as a total number and a percentage.

Abbreviations: SD, standard deviation; REM, rapid eye movements; N1, N2, N3, respectively, sleep stage 1, 2, 3; RERA, respiratory effort related arousals; RDI, respiratory disturbance index; AI, arousal index; AHI, apnoea-hypopnoea index; ODI, oxygen desaturation index; PLMI, periodic limb movements index; PLMAI, periodic limb movements arousal index, NA, not applicable. RDI (higlighted in bold) is the main criteria for OSA diagnosis, as well as one of the main outcomes. ${ }^{a}$ The cutoff of 8 minutes for REM stage latency is chosen according to the diagnostic criteria for narcolepsy.

The high percentage of OSA in our study population is not very surprising as OSA is a very common disorder, with an incidence of $15 \%$ in men and $4 \%$ in women, according to a study from the United States. ${ }^{19}$ This result can also be expected, given that $95 \%$ of our patients suffered from excessive daytime sleepiness and/or tiredness. However, OSA is still under-diagnosed in society, especially among women, and most of our study patients were unaware of their OSA, 
TABLE 4 Distribution of sleep disorders diagnosed on the basis of the PSG findings and questionnaires among the ME/CFS and the non-ME/ CFS groups $(n=78)$

\begin{tabular}{|c|c|c|c|}
\hline Sleep disorder & ME/CFS group $(n=55)$ & Non-ME/CFS group $(n=23)$ & Total $(n=78)$ \\
\hline Non-OSA, PLMD patients & $31(56.4 \%)$ & $9(39.1 \%)$ & $40(51.3 \%)$ \\
\hline Patients with PSG-based diagnosis (OSA, PLMD) & $24(43.6 \%)$ & $14(60.9 \%)$ & $38(48.7 \%)$ \\
\hline OSA: & $20(36.4 \%):$ & $11(47.8 \%):$ & $31(40.3 \%):$ \\
\hline Moderate & $10(50.0 \%)$ & $2(18.2 \%)$ & $12(38.7 \%)$ \\
\hline Severe & $1(5.0 \%)$ & $4(36.4 \%)$ & $5(16.1 \%)$ \\
\hline PLMD & $4(7.3 \%)$ & $3(13.0 \%)$ & $7(8.9 \%)$ \\
\hline OSA + PLMD & $2(3.6 \%)$ & $2(8.7 \%)$ & $4(5.1 \%)$ \\
\hline RLS + PLMD & 0 & $1(4.3 \%)$ & $1(1.3 \%)$ \\
\hline RLS + OSA & $7(12.7 \%)$ & $5(21.7 \%)$ & $12(15.4 \%)$ \\
\hline RLS + PLMD + OSA & $2(3.6 \%)$ & $1(4.3 \%)$ & $3(3.9 \%)$ \\
\hline $\begin{array}{l}\text { More than one sleep disorder diagnosis (from OSA, } \\
\text { PLMD and/or RLS) }\end{array}$ & $36(65.5 \%)$ & $18(78.3 \%)$ & $54(69.3 \%)$ \\
\hline
\end{tabular}

Results presented as number of patients (\%).

Abbreviations: ME/CFS, myalgic encephalomyelitis/chronic fatigue syndrome; OSA, obstructive sleep apnoea; PLMD, periodic limb movement disorder; RLS, restless legs syndrome.

with only a few percent reporting apneas and 18\% snoring. Our remarkably high percentage of women (85\%) points to a selection of patients who suffer from excessive sleepiness/ tiredness together with chronic fatigue that leads to $73 \%$ cases of sick leave, as well as $96 \%$ reporting poor selfreported health, which correlates to increased morbidity and mortality. ${ }^{20,21}$ It is therefore very important to inform society and the healthcare sector that patients complaining of excessive sleepiness/tiredness leading to their inability to work should undergo PSG and sleep questionnaires.

The diagnosis of OSA was made on the basis of the RDI, representing the sum of the AHI and the RERAI, with pathological values in $31(40.3 \%)$ of the patients. However, the ODI values were pathological in only $16(21.1 \%)$ of the patients. This difference means that the breathing pauses caused more sleep fragmentation than oxygen desaturations, which is a sign of a high cortical sensitivity to upper airway obstruction in this patient population. Furthermore, the patients in the present study consisted of $85 \%$ women, mean age 49 years, and $32 \%$ had increased anxiety symptoms and $23 \%$ increased depression symptoms, with a high percentage of poor to very poor self-rated health (96\%). Thus, it seems that patients, and in this cohort especially women, with symptoms of sleepiness/tiredness and high degree of sleep fragmentation are likely to have an increased risk of anxiety, depression and poor self-rated health.

Nearly one half of our patients were diagnosed with RLS. Even though this finding is quite exceptional and stimulates some thoughts about a dopaminergic system dysfunction that can cause restless movements, PLMD, excessive sleepiness and tiredness. We are not, however, able to come to any conclusions because of a very subjective diagnosis of RLS based only on questionnaires and other cofactors such as the medications used.

This PSG-based study revealed that more than half of the chronically fatigued patients had some changes in sleep macrostructure, such as reduced sleep duration, prolonged sleep onset, higher frequencies of arousals and awakenings and more superficial sleep with a longer N1 sleep stage. However, the first limitation of this study is the in-lab, onenight-only investigation using PSG, which could also cause problems with the initiation and maintenance of sleep. The second limitation is that more than half of the participants in our study were using various sleep affecting medications that were not withdrawn prior to the PSG.

Our study points out the previously identified discrepancies in terms of feelings of fatigue, tiredness and sleepiness. Almost all of our 78 chronic fatigue patients complained 
about morning or daytime tiredness, as well as frequent daytime sleepiness. However, the Epworth Sleepiness Scale did only indicate pathological scores in less than half of the sample and did not correlate with the PSG results. Moreover, the Multidimensional Fatigue Inventory subscales were increased (in comparison with normal values in the Swedish population) and did not correlate significantly with objective PSG measures either. ${ }^{17}$ This raises the problems involved in identifying sleep-related disturbances in chronically fatigued patients using the questionnaires used in the present study. However, this is a common problem found in several studies. ${ }^{22,23}$

Self-rated health is known to be a prognostic factor for treatment outcomes, morbidity and mortality. ${ }^{20}$ Almost all patients investigated in this study evaluated their general health as 'poor' or 'very poor'. According to the Canadian CFS diagnostic criteria, in all patients with suspected CFS, the somatic causes of fatigue and illness should be excluded or, in some case, treated. ${ }^{3}$ The negative perception of one's health should indicate that some further diagnostic procedure is necessary in order to find a cause which might be treated in chronically fatigued patients. Moreover, a study conducted by Lekander and colleagues showed that poor subjective health correlates with changes in sleep length and is related to increased fatigue. ${ }^{24}$ However, we did not find any significant relationship between total sleep time and self-rated health in the present study.

Deeper analysis of sleep complaints and use of validated sleep questionnaires should be a part of diagnostic procedure in patients suspected with CFS. PSG should be more frequently used to exclude OSA/PLM. If OSA is found, the adherence of treatment should be followed-up and evaluated by CFS and sleep specialists.

\section{ACKNOWLEDGMENTS}

The authors want to thank Per Julin, $\mathrm{PhD}$, and specialist in rehabilitation medicine, who worked in ME/CFS rehabilitation at Danderyd University Hospital during 2011-2012 for his contribution in initiating this sleep research project in ME/CFS patients.

\section{CONFLICT OF INTEREST}

None.

\section{AUTHOR CONTRIBUTIONS}

Evelina Pajediene analysed data and wrote the paper. Indre Bileviciute-Ljungar designed and performed the study. Danielle Friberg designed and performed the study.

\section{ETHICS}

The use of clinical data for scientific analysis was considered by the regional ethical review board in Stockholm (Ref. no. 2014/300-31) and approved by Danderyd University Hospital (DS2014-0447).

\section{OR CID}

Evelina Pajediene (D) http://orcid.org/0000-0002-6003-4435

\section{REFERENCES}

[1] Skaer TL, Sclar DA. Economic implications of sleep disorders. Pharmacoeconomics. 2010;28(11):1015-1023.

[2] Fukuda K, Straus SE, Hickie I, et al. The chronic fatigue syndrome: a comprehensive approach to its definition and study. Ann Intern Med. 1994;121(12):953-959.

[3] Carruthers B, Jain A, De Meirleir K, et al. Myalgic encephalomyelitis/chronic fatigue syndrome: clinical working case definition, diagnostic and treatment guidelines: a consensus document. J Chron Fat Syndr. 2003;11:7-115.

[4] Nacul LC, Lacerda EM, Pheby D, et al. Prevalence of myalgic encephalomyelitis/chronic fatigue syndrome (ME/CFS) in three regions of England: a repeated cross-sectional study in primary care. BMC Med. 2011;9:91

[5] Mariman A, Vogelaers D, Tobback E, et al. Sleep in the chronic fatigue syndrome. Sleep Med Rev. 2013;17:193e199.

[6] Unger ER, Nisenbaum R, Moldofsky H, et al. Sleep assessment in a population-based study of chronic fatigue syndrome. $B M C$ Neurol. 2004;4:6

[7] Myers C, Wilks D. Comparison of Euroqol EQ-5D and SF-36 in patients with chronic fatigue syndrome. Qual Life Res. 1999;8 (1e2):9e16

[8] Mariman A, Vogelaers D, Hanoulle I, et al. Subjective sleep quality and daytime sleepiness in a large sample of patients with chronic fatigue syndrome (CFS). Acta Clin Belg. 2012;67(1): $19 \mathrm{e} 24$

[9] Mariman A, Delesie L, Tobback E, et al. Undiagnosed and comorbid disorders in patients with presumed chronic fatigue syndrome. J Psychosom Res. 2013;75(5):491-496.

[10] Neu D, Mairesse O, Montana X, et al. Dimensions of pure chronic fatigue: psychophysical, cognitive and biological correlates in the chronic fatigue syndrome. Eur J Appl Physiol. 2014; 114(9):1841-1851

[11] Johns MW. A new method for measuring daytime sleepiness: the Epworth Sleepiness Scale. Sleep. 1991;50-55.

[12] Walters AS, LeBrocq C, Dhar A, et al. International Restless Legs Syndrome Study Group. Validation of the International Restless Legs Syndrome Study Group rating scale for restless legs syndrome. Sleep Med. 2003;4(2):121-132.

[13] Benyamin Y. Why does self-rated health predict mortality? An update on current knowledge and a research agenda for psychologists. Psychol Health. 2011;26:1407-1413.

[14] Smets EM, Garssen B, Bonke B, et al. The Multidimensional Fatigue Inventory (MFI) psychometric qualities of an instrument to assess fatigue. J Psychosom Res. 1995;39(3):315-325. 
[15] Zigmond AS, Snaith RP. The hospital anxiety and depression scale. Acta Psychiatr Scand. 1983;67:361-370.

[16] Iber C, Ancoli-Israel S, Chesson AL, Jr, et al. The AASM Manual for the Scoring of Sleep and Associated Events: Rules, Terminology and Technical Specifications. Westchester, IL: American Academy of Sleep Medicine; 2007.

[17] Ericsson A, Mannerkorpi K. Assessment of fatigue in patients with fibromyalgia and chronic widespread pain. Reliability and validity of the Swedish version of the MFI-20. Disab Rehab. 2007;29:1665-1670.

[18] Le Bon O, Fischler B, Hoffmann G, et al. How significant are primary sleep disorders and sleepiness in the chronic fatigue syndrome? Sleep Res Online. 2000;3(2):43-48.

[19] Peppard PE, Young T, Barnet JH, et al. Increased prevalence of sleep-disordered breathing in adults. Am J Epidemiol. 2013;177 (9):1006-1014.

[20] Gill TM, Feinstein AR. A critical appraisal of the quality of quality of life measurements. JAMA. 1994;272:619-626.

[21] Nerfeldt P, Aoki F, Friberg D. Polygraphy vs. polysomnography: missing OSAS in symptomatic snorers - a reminder for clinicians. Sleep Breath. 2014;18(2):297-303.
[22] Macey PM, Woo MA, Kumar R, et al. Relationship between obstructive sleep apnea severity and sleep, depression and anxiety symptoms in newly-diagnosed patients. PLoS One. 2010;5 (4):e10211.

[23] Johansson P, Alehagen U, Svanborg E, et al. Sleep disordered breathing in an elderly community-living population: relationship to cardiac function, insomnia symptoms and daytime sleepiness. Sleep Med. 2009;10(9):1005-1011.

[24] Lekander M, Andreasson AN, Kecklund G, et al. Subjective health perception in healthy young men changes in response to experimentally restricted sleep and subsequent recovery sleep. Brain Behav Immun. 2013;34:43-46.

How to cite this article: Pajediene E, BileviciuteLjungar I, Friberg D. Sleep patterns among patients with chronic fatigue: A polysomnography-based study. Clin Respir J. 2018;12:1389-1397. https://doi.org/10. $\underline{1111 / \text { crj. } 12667}$ 